\title{
INTRODUCCION AL ESTUDIO DE LOS HONGOS SUPERIORES IV.
}

\author{
(Introduction to the study of higher fungi IV.)
}

\author{
Waldo Lazo A. \\ Departamento de Ciencias Ecológicas, \\ Facultad de Ciencias, Universidad de Chile, \\ Casilla 653 - Santiago, Chile.
}

Palabras clave: Ascomycetes, Basidiomycetes

Key words: Ascomycetcs, Basidiomyceics

\section{RESUMEN}

Se presentan descripciones e ilustraciones en color de 12 hongos comunes de Chile: el Ascomycete Sarcoscypha coccinea y los Basidiomycetes Thelephora terrestris, Trametes versicolor, Ganoderma australe, Stereum hirsutum, Suillus luteus, Volvariella speciosa, Psilocybe semilanceata, Panaeolus retirugis, Heheloma mudipes, Setchelliogaster tenuipes, Cyathus stercoreus.

Debido a la falta de una obra con ilustraciones en color de los hongos superiores de Chile, Lazo, (1984 - 1996) dio a conocer datos morfológicos y biogeográficos de $6+$ especies fúngicas (y sus ilustraciones). muchas de ellas no siempre fáciles de encontrar. Continuando con csla labor, se describen doce hongos comunes en nucstro país incluidas cn los siguientes órdenes: Pexizales, Thelepho rales, Stereales, Poriales, Ganodermatales, Boletales, Agaricales, Cortinariales y Nidulariales y se presentan fotografias de sus estructuras reproductivas. Al igual que en las publicaciones antes citadas, los textos e ilustraciones, forman parte de la obra "Hongos de Chile. Atlas Micológico" (Lazo, inćdito).

Sarcoscypha coccinea (Fr.) Lambolte in Mem. Soc. Roy. Sciences. Licge II. 14: 303. 1997

Apolccios de 15-36 mm de diámetro, cupuliformes, estipitados, con el margen ligeramente incurvado. a veces algo dentado y amarillento, la superficic externa es blanca y flocosa. Himenio rojo cscarlata. Estípite de largo variable, a veces de más de $30 \mathrm{~mm}$. lijeramente velloso. Esporas

\section{SUMMARY}

Descriptions and color illustrations of 12 common Chilean fungi are presented: the Ascomycete Sarcoscypha coccinea and the Basidiomycetes Thelephora terrestris, Trametes versicolor, Ganoderma australe, Stereum hirsutum, Suillus luteus, Volvariella speciosa, Psilocybe semilanceata, Panaeolus retirugis, Hebeloma nudipes, Setchelliogaster tenuipes, Cyathus stercoreus.

de 28-36 x 12-13 $\mu \mathrm{m}$, clíptico cilíndricas con los extremos obtusos, hialinas, con gútulas. Ascos de 400-450 × 14-15 $\mu \mathrm{m}$, octosporados, cilíndricos-adelgazados en la base. Parafisis ramificadas, delgadas, con contenido granular de color rojo y con las puntas aguzadas. Habitat: en palos semicnterrados, en cl bosque. Especie no comestible. Distribución: muy abundante en Chile central desde junio a octubre.

\section{Thelephora terrestris Fr., Syst. Myc. 1: 431. 1821}

Basidiocarpo de $50-80 \mathrm{~mm}$ de alto, ferruginoso a pardo, margen blanco, dimidiado, efuso-reflejado, a veces en forma de roseta. Himenio de color pardo chocolate a pardo violácco, arrugado o granular. Esporas de $10 \times 6-9 \mu \mathrm{m}$, fuscas, elipsoides, cquinuladas. Hifas con fibulas. Habitat: basidiocarpos congregados en el suelo en los bosques de Pinus spp. Especie no comestible. Distribución: Chilc central y austral.

Stereum hirsutum (Willd.) Pers. ex. Gray, Nat. Arr. British Plants 1:652. 1821 
Basidiocarpo de 20-70 $\mathrm{mm}$ de ancho, efusado, con la cara superior amarillo anaranjada a rojiza, grisácea, zonada, velludo-cstrigosa, con el margen amarillo. Cara inferior (himenial) amarillo anaranjada a grisácca, lisa, parcja. Contextura amarillenta, coriácea, firme y resistente. Esporas hialinas de 6-8 $\times 3$ 3-3.6 $\mu \mathrm{m}$, elíptico oblongas. No hay cistidios. Habitat: sobre postes, tocones, troncos y ramas caídas. Especie no comestible. Distriluución: Chile central y austral.

Trametes versicolor (L. ex Fr.) Pilat, Aul. Polyp., p.261. 1939

Pílco de 60-250 mm, ocre grisáceo con zonas multicolores de colores llamativos, aplanado, atcrciopelado, sésil. Poros de 0.3-0.4 $\mathrm{mm}$ de anchura, blancos a amarillentos. Contextura blanquizca, recia, coriácea. Tubos cortos. Esporas de $6-8 \times 2-3 \mu \mathrm{m}$, cilíndricas, lisas, deprimidas. Basidios de 14-17 × 5-6 $\mu \mathrm{m}$, bi o tetrasporados. Habitat: en madera. Especie no comestible. Distrilución: Chile central y austral.

Ganoderma australe (Fr.) Pat., Bull. Soc. Myc. 5: 71. 1889

Píleo de 220-320 mm de anchura, pardo oscuro con algunas zonas grises, semiorbicular aplanado, convexo, a veces ondeado, dimidiado, sésil. Superficie cubierta por una costra lacada, brillante, gruesa, muy dura y que con posterioridad se resquebraja. Margen glabro, estrecho, estéril. Tubos de $25-30 \mathrm{~mm}$ de profundidad, fuscos, desprendibles a veces. Poros de $200 \mu \mathrm{m}$ de ancho, blancos al principio, después pardos. Contextura parda obscura, recia, fibrosa. Esporas de 9-10 $\times 7 \mu \mathrm{m}$, parduzcas, clípticas, truncadas. Hifas parduzco pálidas, con paredes gruesas. Hablitat: parásito de diferentes árboles. Especie no comestible. Distribución: Chile central y austral.

Suillus luteus (L. ex Fr.) Gray. Nat Arr. Brit. Pl. 1: 646. 1821

Píleo de 40-140 $\mathrm{mm}$ de diámetro, parduzco o rojizo parduzco a castaño ahumado, glutinoso, liso, con la película facilmente removible. Tubos de 5-8 $\times 1 \mathrm{~mm}$, de color amarillo pálido cuando nucvos, con posterioridad ocráceos u oscuros, angulares e irregulares, cubiertos al principio por un velo blanco. Estípite de 65-95 $\times$ 7-12 $\mathrm{mm}$, amarillento u oscuro arriba, blanquizco hacia la base, punteado, parejo, Velo: membrana blanca que forma un anillo colapsado a $10 \mathrm{~mm}$ del extremo superior del estípite. Contextura de 12-15 mm de grosor, de color crema, recia y elástica, con la película muy ácida. Sabor agradable. Esporada pardo dorada. Esporas de 7.5-9.5 x 3-3.7 $\mu \mathrm{m}$, pardas, lisas, elípticas. Habitat: se desarrolla en el suelo, especialmente en los bosques de Pinus spp con los que forma micorrizas. Hongo comestible y de mucha aceptación. Distribución: Chile central y austral.

Volvariella speciosa (Fr. ex. Fr.) Sing., Lilloa 22:401. 1951
Pílco de 50-120 mm de diámetro, de color blanco sucio a grisáceo, globoso-aovado al principio, después expandido a plano, a veces ligeramente cóncavo, y con un pequeño umbo, viscoso, glabro. Lamelas rosadas, libres, anchas, congregadas. Estípitc de 100-150 × 10-20 mm, blanco sucio, glabro, parcjo, algo bulboso en la base, sólido. Volva blanca, grande, flácida, algo lobulada. Contextura delgada, suave, putrescente, olor marcado. Sabor discreto. Esporada rosada. Esporas de 14-19.5 x 7.7-10.8 $\mu \mathrm{m}$, ligeramente rosadas, lisas elipsoides. Basidios de $36-48 x$ $14 \mu \mathrm{m}$, tetrasporados. Cistidios hialinos, fusoides, anchos. Epicutis gelatinizado. Habitat : se desarrolla en el suelo, en los potreros entre el pasto. Especie comestible. Distribución: Chile central y austral.

Panaeolus retirugis (Fr.) Gillet, Hymenom. :621. 1874

Pílco de 15-18 mm de diámetro, de color ante a ocráceo, campanulado cónico, margen apendiculado. Lamelas grisáceo negruzcas, variegadas, adnado ad-nexadas, con borde blanco. Estípite de 35-40 × 3-4 mm, de color ante, parejo, sólido. Esporada negra. Esporas de 11-13 $\times 8 \times 7 \mu \mathrm{m}$, negruzcas, lentiformes, con poro germinativo. Basidios de 25-32 x 9-12 $\mu \mathrm{m}$. Hay queilocistidios. Hifas con fíbulas. Habitat: sobre estićrcol. Hongo tóxico. Distribución: Chile central y austral.

Psilocybe semilanceata (Fr. ex Secr.) Kummer, Fuhr. Pilzsk:71. 1871

Pílco de 5-12 mm de diámetro, y 15-20 mm de altura ocráceo parduzco, cónico con una papila central bastante pronunciada. Lamelas ascendentes, de color argiláceo, ventricosas. Estípite de 7()-100 × $2 \mathrm{~mm}$, blanco méleo, más oscuro cerca de la base, liso, levemente adelgazado hacia la punta. Esporada parda. Esporas de 12.5-16 $\times 7.5-9 \mu \mathrm{m}$ pardo liláceas en agua, lisas, con un amplio poro germinal truncado. Basidios tetrasporados. No hay pleurocistidios, queilocistidios vesiculosos, ventricosos. Hifas con fibulas. Habitat: en suelos con estiércol equino o bovino entre el pasto. Hongo tóxico, alucinógeno. Distribución: Chile central y austral.

Hebeloma nudipes (Fr.) ss. Kühn. et Romagn, Fi. Analytique Champignon Sup. 247. 1953

Pílco de 40-70 mun de diámetro de color castaño pálido a crema parduzco, convero, después aplanado, obtuso, desigual, liso, viscoso cuando está mojado, sedoso brillante cuando está seco, con cl margen membranácco. Lamelas de color castaño oscuro, juntas, adnado adnexadas. Estípitc de 50-80 × 8-16 mm, blanco crema, parejo, liso arriba, fibriloso en la base, recto. Contextura blanca carnosa. Esporada parda oscura. Olor ligero. Sabor suave. Esporas de 12-15 × 6-7 $\mu \mathrm{m}$, fuscas, elípticas. Habitat: se desarrolla en el suelo entre las agujas secas de los bos- 

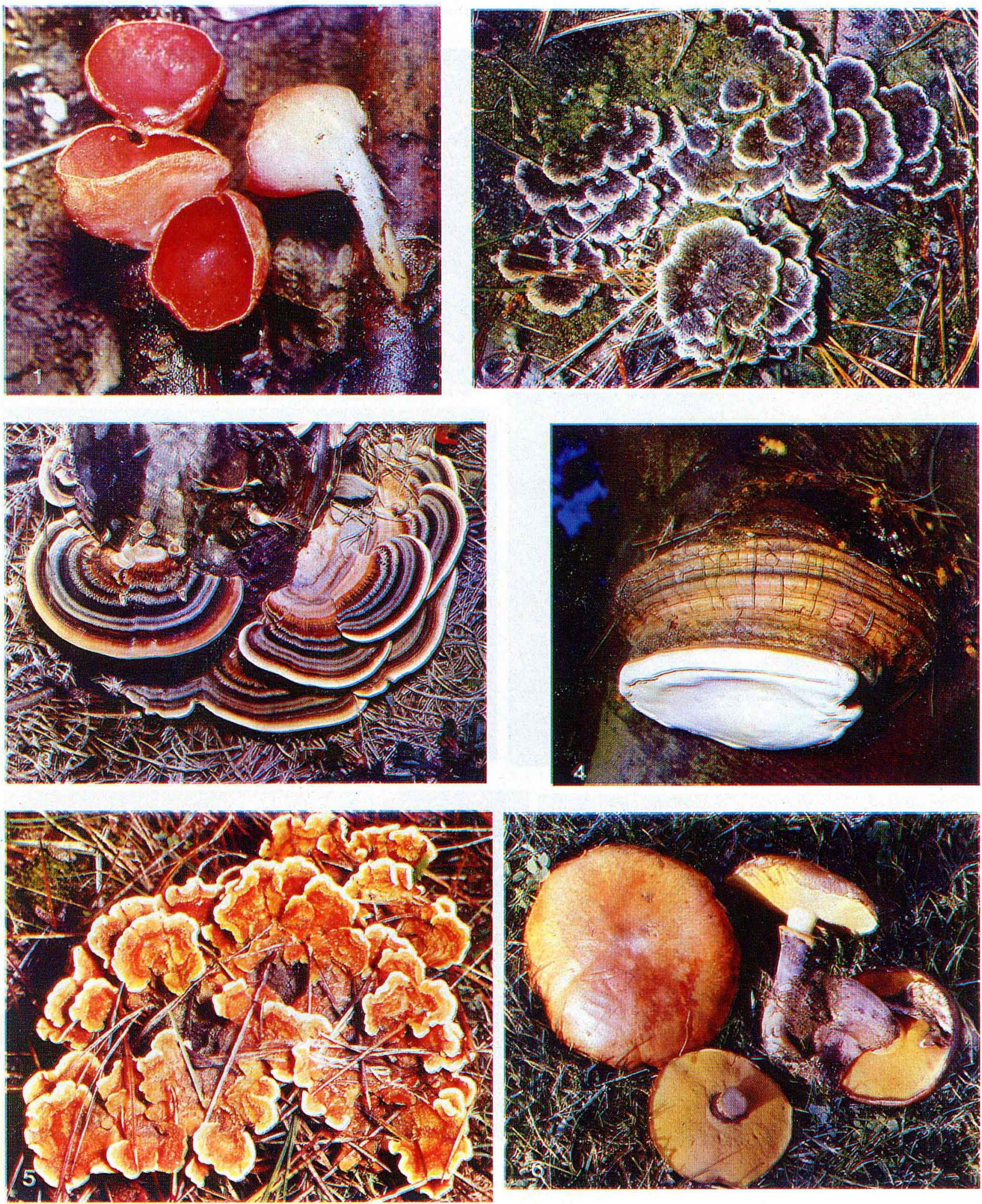

Figuras. 1-6.- 1. Sarcoscypha coccinea 2.- Thlephora terrestris 3. Trametes versicolor 4. Ganoderma australe 5. Stereum hirsutum 6. Suillus luteus 

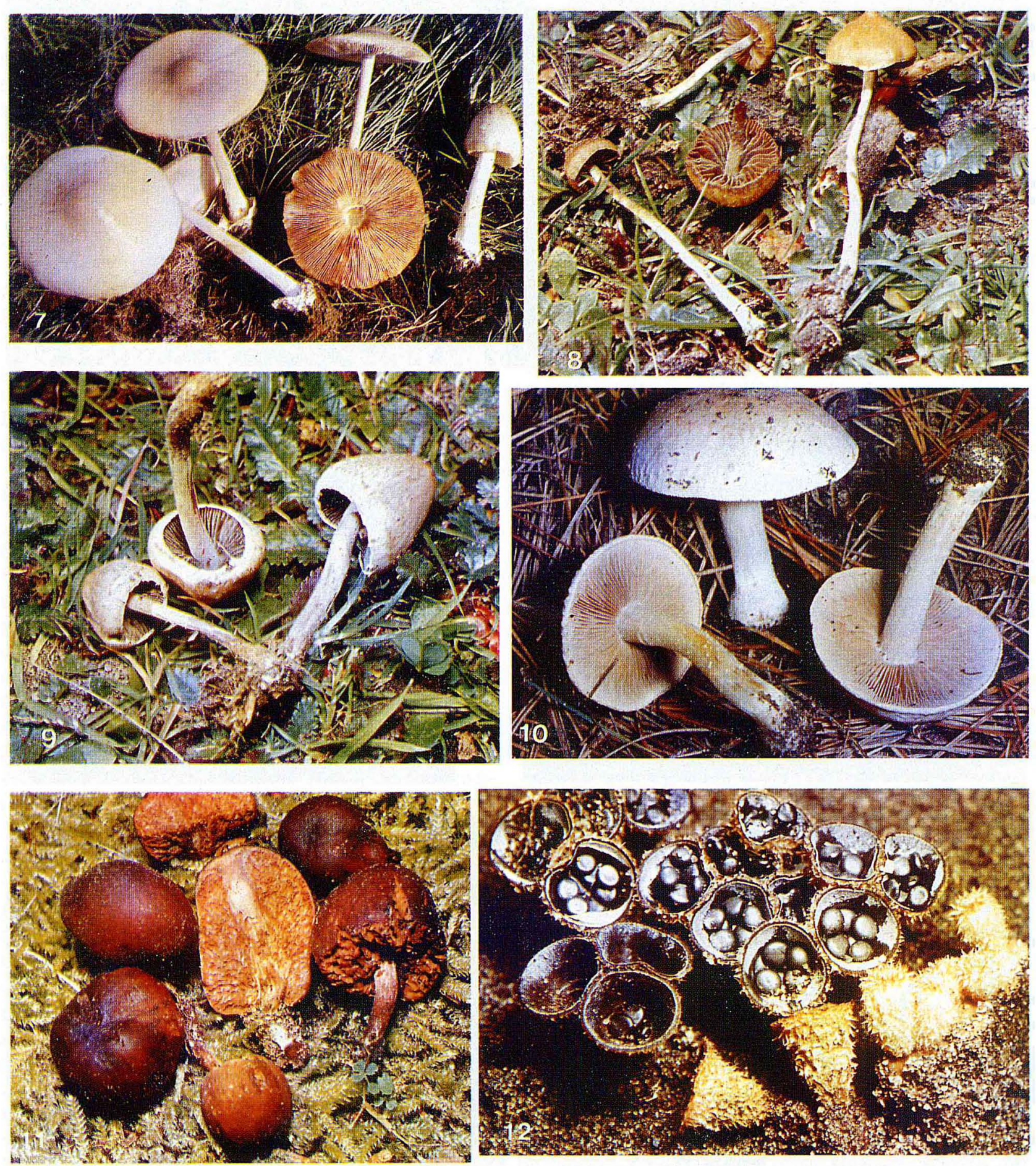

Figuras ( 7-12). 7. Volvariella speciosa 8. Psilocybe semilanceata 9. Pnaneolus retirugis 10. Hebeloma nupides 11. Setchelliogaster tenuipes 12. Cyathus stercoreus 
ques de pinos. Especie no comestible. Distribución: Chile central y austral.

Garrido (1985) anota como nombre válido para csta especic: Hebeloma longicaudum Fr. ss. Lange.

Setchelliogaster temuipes (Sctchcll) Pouzar, Ceská Mykologic 12:3+. Figs. 1-3. 1958

Gastrocarpo de 12-32 $\times 10-28 \mathrm{~mm}$. pardo rojizo a color ladrillo, subgloboso a ovoide. truncado en la base. Peridio pardo rojizo. glabro. seco. Gleba ocrácco parda, loculada. lameloide en algunos cjemplares. Estípite de $10-22 \times 2-3 \mathrm{~mm}$. concoloro con el pílco y más pálido $\mathrm{en}$ la parte superior. Columela continua con el estípite y ensanchada en la parte superior al llegar al peridio. No hay volva. Olor muy aromático y que recucrda algo el del suclo mojado. Esporas de 13-17.2 × 8.6-10.8 $\mu \mathrm{m}$, pardo roya, elipsoides a sub-ovoides. Exosporio consistente de una pared contínua a trarćs de la cual se extienden pequeños canales taponados por una sustancia resinosa dorada. Basidios de 32-37 $\times 8-10 \mu \mathrm{m}$, hialinos, gencralmente bisporados. Habitat: en cl suclo entre cl pasto o los mus- gos en bosques de Eucalyptus sp. y Pinus radiata. Especie no comestible. Distribución: Chile central. California. Orcgonl.

Cy'athus stercoreus (Schw.) De Toni in Sacc. Syll. Fung.. VII 1:4(). 1888

Gist rocarpo de 8-12 $\mathrm{mm}$ de altura y 6-7 $\mathrm{mm}$ de ancho en cl ápice. con forma de vaso y adelgazado hacia la base en la cual su diámetro es sólo de $1.5 \mathrm{~mm}$. sésil. Superficie externa de color ante a ligeramente parduzca, cubierta con vellos gruesos y lanudos, al cnvejecer toma un tinte gris y llega a ser lisa. Borde entero y parcjo. Superficie interna negruzca, lisa, algo brillante. Epifragma más bien fugaz. Peridiolos de 2-2.5 mm de ancho, negruzcos, lenticulares, brillantes, carentes de una túnica. Funículo corto. Esporas de $32-30 \mu \mathrm{m}$, hialinas, subglosas, lisas. Habitat: en suclos donde hay estiércol de vacunos. Muy común en los potreros donde pastan los vacunos. También a veces en los invernaderos. Especie no comestible. Distribución: Chile central y austral.

\section{REFERENCIAS}

Garrido, N. (1985). Index Agaricalium Chilensium. I. Cramer. Vaduz.

Lazo, W. (1984). Introdución al estudio de los hongos superiores III. Bol. Micol. 2:27-66

(1996). Algunos Ascomycetes y Basidiomycetes interesantes de Chile. Bol. Micol. 11:99-102
Singer, R. (1969). Mycollora Australis. Bcilh. Nora Hedwigia 29:1406

Spegaraini, C. (1910). Fungi Chilensis. Contribución al estudio de los hongos chilenos. Libreria Nacional. Buenos Aires.

$25: 1-124$
(1921). Mycetes Chilensis. Bol. Acad. Nat. Cs. (inédito). Hongos de Chile. Atlas Micológico. 